\title{
A Hybrid Method for Evaluating Biomass Suppliers - Use of Intuitionistic Fuzzy Sets and Multi-Periodic Optimization
}

\author{
Vassilis C. Gerogiannis, Vasiliki Kazantzi, and Leonidas Anthopoulos \\ Project Management Department, \\ Technological Education Institute of Larissa, 41110, Larissa, Hellas \\ \{gerogian, kazantzi, lanthopo\} ateilar.gr
}

\begin{abstract}
Evaluation of biomass suppliers is a time-dependent problem that requires assessment of different supply schemes in different periods. This paper presents a hybrid method for evaluating biomass suppliers that combines Intuitionistic Fuzzy Sets (IFS), linear programming (LP) and multi-periodic optimization (MPO). IFS allow evaluators to express their hesitation when they assess alternative suppliers. LP is used to estimate weights of evaluation criteria and calculate suppliers' ratings in a specific period. These ratings are utilized by a MPO model to determine what type and how much feedstock should be supplied by each supplier in each period.
\end{abstract}

Keywords: Biomass Supplier Evaluation, Intuitionistic Fuzzy Sets, MultiPeriodic Optimization.

\section{Introduction}

One way to deal with the seasonality of biomass supply requirements and achieve cost-effective supplies is via procurement from different suppliers in different time periods [1]. Alternative suppliers need to be evaluated regularly because of the timedependency of most of the evaluation criteria. The problem is also characterized by high uncertainty [2] because of subjective judgments on biomass quality aspects, unexpected demand, variable raw material prices, variation in biomass availability and unstable procurement lead times.

The work presented in this paper emphasizes on evaluating biomass suppliers to maximize the total benefit for the supply chain and minimize the associated production and logistics costs. A multi-criteria method is suggested for supporting biomass supplier evaluators to solve two practical problems: (i) What is the optimal combination of biomass suppliers that can provide the required quantities of raw materials for bioenergy production to a conversion plant in certain periods of time? (ii) How much of the required biomass materials should be purchased from each supplier in each time period? The presented method addresses evaluators' judgments with Intuitionistic Fuzzy Sets (IFS) [3]. IFS consider, not only the uncertainty of evaluators to quantify (qualitative) evaluation criteria and determine suppliers' ratings on the identified criteria, but also evaluators' positive and negative judgments, which 
need not to be complementary. IFS include membership and non-membership of an element to a fuzzy set, as well as a third parameter that is called the hesitation degree. Expression of indeterminacy can be suitable to evaluate suppliers in a highly uncertain supply network, such as a biomass supply system. An IFS-based technique [4] is applied in combination with linear programming to derive weights for the evaluation criteria and suppliers' ratings. The technique results (supplier ratings) are further utilized by a multi-periodic optimization model that is proposed to determine what type and how much feedstock should be supplied, in each period, from each supplier.

\section{Biomass Supplier Evaluation Criteria and Supplier Selection Methods}

From reviewing the relevant literature, a set of criteria were identified for the evaluation of biomass suppliers [1] [2]: (i) Reliability. It includes, as sub-criteria, adherence to contract terms, ensuring on-time delivery and agreed biomass quality. Suppliers need to provide proper feedstock, at correct amounts, within right times, and at right conditions. (ii) Responsiveness. Unexpected demand variations may cause operational problems in bioenergy production. Biomass availability and seasonality are time-dependent characteristics that can lead to supply chain malfunctioning. (iii) Flexibility. In biomass systems, flexibility refers to the ability of a supplier to provide alternative biomass types that match the needs of the production process and facilitate uninterrupted biomass flow. (iv) Cost. Biomass market prices can be viewed as unstable parameters determined by changes in aggregate demand and supply conditions. (v) Quality aspects. Variability in quality of feedstock types is usually an undesirable aspect of provided sources. Quality aspects include biomass moisture, density and energy content, quality compliance, defect rates and quality certification possession. (vi) Assets and infrastructure. The economic performance of a supply chain is affected by the relative location of the biomass production site and existing transportation infrastructure. This category also includes facility and fleet size, warehouses number and capacity. (vii) Environment and safety. These criteria are related with a supplier's consideration to environmental and safety issues.

Most of these criteria are qualitative and unstable in nature and, therefore, a fuzzybased multi-criteria decision making (MCDM) method can be proven beneficial for their characterization [5]. In the category of fuzzy-based MCDM, two classes of methods can be broadly identified. In the first class, there are methods which handle imprecise evaluation criteria and supplier ratings with fuzzy numbers, while in the second calls there methods making use of linguistic terms to evaluate criteria and suppliers. There are also hybrid methods combining fuzzy logic with techniques such as the Analytic Hierarchy Process - AHP [6] and the Technique for Order Performance by Similarity to Ideal Solution - TOPSIS [7]. Furthermore, some generalizations of fuzzy sets are valuable to deal with indeterminacy in a supplier selection setting. Such an extension is Intuitionistic Fuzzy Sets (IFS) [3] which allow evaluators to express hesitation degree or ambiguity, when they assess alternative suppliers and criteria weights. Representative examples of IFS applications in supplier 
selection problems can be found in [8] [9]. In the next section, we adopt and extend an IFS-based technique that was originally suggested by Li [4]. The main reason to utilize this technique in a biomass supplier evaluation setting is that it provides a comprehensive way to calculate optimal weights for the evaluation criteria, in each time period considered. The technique can be repeatedly applied for each period, to derive new criteria weights and new supplier ratings based on these weights. We present how this technique can further extended by using its results as inputs to a linear, multi-periodic optimization model. The final result is the optimal combination of suppliers with their respective optimal types and amounts of biomass supplies for each time period considered.

\section{Description of the Method}

Assume that there is a set of $n$ biomass suppliers $S=\left\{S_{1}, S_{2}, \ldots, S_{n}\right\}$. Each supplier has to be evaluated on $m$ evaluation criteria $X=\left\{X_{1}, X_{2}, \ldots, X_{m}\right\}$. There is also a group of $k$ evaluators responsible to evaluate the suppliers, in each time period. In the first step of the method, the values of $\mu_{i j}, v_{i j}$ and $\pi_{i j}$ are determined which specify, respectively, the degree of membership, non-membership and hesitation for a supplier $S_{j}$ with respect to criterion $X_{i}$. These degrees represent the evaluation of the fuzzy concept "appropriateness of $S_{j}$ offer with respect to $X_{i}$ ". One way to derive these values is by asking all $k$ evaluators to express their judgment whether $S_{j}$ offer is appropriate or not to fulfill criterion $X_{i}$. Suppose that from the $k$ evaluators, $k_{1}$ consider $S_{j}$ offer is strong, $k_{2}$ express that $S_{j}$ offer is weak and $k_{3}$ give no answer, due to their indeterminacy $\left(k=k_{1}+k_{2}+k_{3}\right)$. Then, $\mu_{i j}, v_{i j}$ and $\pi_{i j}$ are calculated as follows:

$$
\mu_{i j}=k_{1} / k, v_{i j}=k_{2} / k, \pi_{i j}=k_{3} / k
$$

Given the indeterminacy of evaluators, a hesitation in the degree of $\mu_{i j}$ exists, denoted by a lower $\mu_{i j}^{l}$ and an upper bound $\mu_{i j}^{u}$ which are expressed as follows:

$$
\mu_{i j}^{l}=\mu_{i j}, \mu_{i j}^{u}=\mu_{i j}+\pi_{i j}=1-v_{i j}
$$

The second step is to determine the weight of each criterion by asking each evaluator to assess the criteria with respect to their impact on the production. Each evaluator compares the criteria pair-wise (by following AHP) and $k$ weights are derived, for each one of the $m$ criteria. Each weight $\omega_{i}$ is a number that lies in the interval $\left[\omega_{i}^{l}, \omega_{i}^{u}\right]$, where $\omega_{i}^{l}$ is the minimum and $\omega_{i}^{u}$ is the maximum weight for criterion $X_{i}$. To determine optimal weights, the following optimization model can be solved (eq. (3)) [4]: 


$$
\max \left\{\frac{\sum_{j=1}^{n} \sum_{i=1}^{m}\left(\mu_{i j}^{u}-\mu_{i j}^{l}\right) \omega_{i}}{n}\right\} \text {, subject to: } \omega_{i}^{l} \leq \omega_{i} \leq \omega_{i}^{u} \forall i=1, \ldots, m, \sum_{i=1}^{m} \omega_{i}=1
$$

The computed weights can be utilized to calculate lower and upper bounds of the weighted rating for each supplier based on eqs. (4) \& (5):

$$
\begin{gathered}
z_{j}^{l}=\sum_{i=1}^{m} \mu_{i j}^{l} \omega_{i}=\sum_{i=1}^{m} \mu_{i j} \omega_{i} \forall j=1, \ldots, n \\
z_{j}^{u}=\sum_{i=1}^{m} \mu_{i j}^{u} \omega_{i}=1-\sum_{i=1}^{m} v_{i j} \omega_{i} \forall j=1, \ldots, n
\end{gathered}
$$

To obtain the final rating of supplier $S_{j}$, a comparison index $\xi_{j}$ based on the TOPSIS method is calculated, as follows (eq. (6)):

$$
\xi_{j}=\frac{D\left(A_{j}^{0}, B\right)}{D\left(A_{j}^{0}, B\right)+D\left(A_{j}^{0}, G\right)}
$$

In eq. (6), $A_{j}^{0}, G$ and $B$ represent the optimal rating for supplier $S_{j}$, the ideal alternative supplier and the negative ideal alternative supplier respectively. $D$ stands for the Hamming Distance measure, as it was defined for IFS [10]. By using the Hamming Distance, the comparison index $\xi_{j}$ can be computed as shown in eq. (9).

$$
\begin{gathered}
A_{j}^{0}=\left\{<S_{j}, z_{j}^{l}, 1-z_{j}^{u}>\right\}=\left\{<S_{j}, \sum_{i=1}^{m} \mu_{i j} \omega_{i}, \sum_{i=1}^{m} v_{i j} \omega_{i}>\right\} \\
G=\{<g, 1,0>\} \text { and } B=\{<b, 0,1>\} \\
\xi_{j}=\frac{z_{j}^{u}}{1+z_{j}^{u}-z_{j}^{l}}
\end{gathered}
$$

The ranking derived for each supplier can be consequently utilized to answer the following questions: Which suppliers should be finally chosen based on the derived ratings and when? What type of and how much feedstock should be supplied by each supplier? How would total budget for the biomass purchasing function be allocated? What would be the maximum total purchasing value in each period? To answer these questions, we consider a decision-making horizon $H$, during which variations in market conditions are anticipated in terms of time-dependent changes in biomass quantities, types and prices of supply and demand. $H$ is discretised into $N t$ periods (i.e., within each period purchasing conditions are assumed to be stable). By applying repeatedly the previous steps of the IFS-based technique, suppliers' ratings can be 
calculated per time period. An optimization model, using the suppliers' ratings as inputs (coefficients) of the objective function, can be specified, as follows.

Objective function:

$$
\max \left(T P V_{t}\right)=\sum_{b \in B} \sum_{j b \in J_{b}} w_{t, j} F_{t, j_{b}} \quad \forall t \in T
$$

where: $T P V_{t}=$ Total Purchasing Value in period $t, j=$ supplier index, $b=$ biomass type, $j_{b}=$ index of supplier $j$ providing biomass type $b, w_{t, j}=$ rating of supplier $j$ in period $t$, $F_{t, j b}=$ amount of biomass type $b$ to be delivered from supplier $j$ in period $t, J_{b}=$ set of suppliers delivering feedstock type $b, B=$ set of feedstock types and $T=$ set of periods.

Capacity constraints: $\quad F_{t, j_{b}} \leq F_{t, j_{b}}^{\max } \quad \forall t \in T, \forall j_{b} \in J_{b}, \forall b \in B$

Demand Constraints: In each period $t$, the procured amounts of all biomass types $b$ from all suppliers providing biomass $b$ must sum up to the demand for feedstock, $D_{t}$ :

$$
\sum_{b \in B} \sum_{j_{b} \in J_{b}} F_{t, j_{b}} \leq D_{t} \quad \forall t \in T
$$

Budget constraints: $\quad \sum_{b \in B} \sum_{j_{b} \in J_{b}} F_{t, j_{b}} c_{t, j_{b}} \leq C_{t}^{\max } \quad \forall t \in T$

$C_{t}^{\text {max }}$ refers to the total purchasing budget in period $t$ and $c_{t, j_{b}}$ is the total cost for purchasing biomass type $b$ from supplier $j$ in period $t$.

Quality Constraints (optional): Quality constraints ensure that required production quality levels are maintained.

$$
\sum_{j_{b} \in J_{b}} F_{t, j_{b}} q_{t, j_{b}} \leq D_{t} Q_{t, b} \quad \forall t \in T, \forall b \in B
$$

$Q_{t, b}$ is the buyer's maximum acceptable defect rates for each biomass type $b$ in period $t$ and $q_{t, j_{b}}$ is the defect rate of supplier $j$ with respect to biomass type $b$ at period $t$.

Non negativity constraints: $\quad F_{t, j_{b}} \geq 0 \quad \forall t \in T, \forall j_{b} \in J_{b}, \forall b \in B$

To validate the method, we have considered a case problem of a bioenergy production plant in which three suppliers $S_{1}, S_{2}$ and $S_{3}$, offer specific biomass types; $S_{1}$ provides rapeseed (RP), $S_{2}$ provides both rapeseed (RP) and sunflower (SN) and $S_{3}$ delivers waste cooking oil (WCO). By using repeatedly the IFS-based technique (eqs. (1)-(9)), ratings of the three suppliers were calculated on a monthly basis. Capacity constraints for each supplier per month were also considered. If the total budget is $9000 €$, total demand is $1000 \mathrm{tn} / \mathrm{month}$ and purchasing costs are $5.5 € /$ tn for $S_{1}, 8 € / \mathrm{tn}$ for $S_{2}$ and $6.5 € /$ th for $S_{3}$, the multi-periodic optimization problem was repeatedly solved (per month) by using eqs. (10)-(15). Suppliers' selection profiles and biomass 
amounts are depicted in Figure 1. Supplier $S_{2}$ and $S_{3}$ compete for being the best alternatives. This finding can be justified since $S_{3}$ represents a low-cost supply alternative (providing inexpensive WCO), whereas $S_{2}$ exhibits flexibility by offering adequate feedstock amounts of two biomass types $(b 1=\mathrm{RR}$ and $b 2=\mathrm{SN})$.

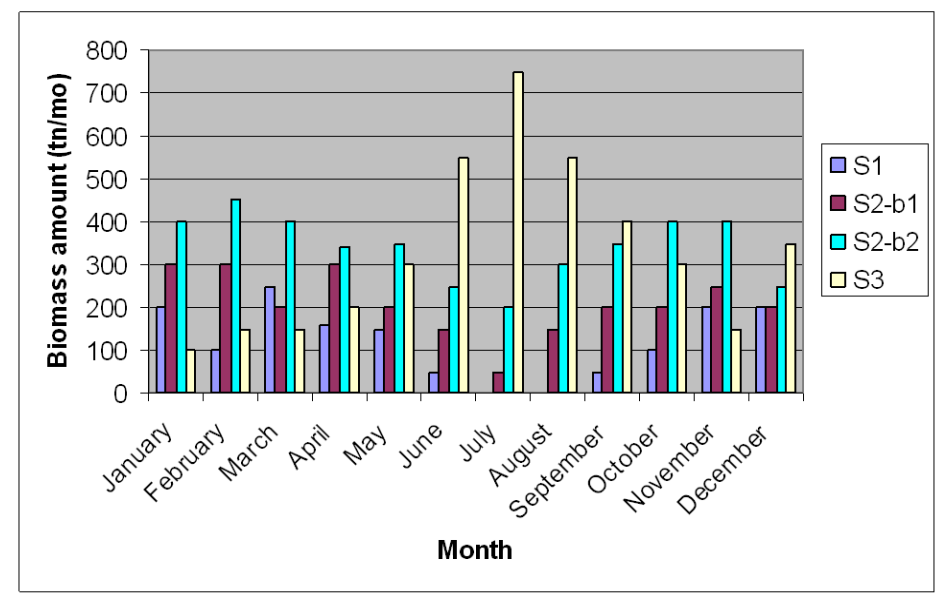

Fig. 1. Optimal supply profiles and purchased biomass schedule per month

\section{Conclusions}

The paper presented a multi-criteria decision making method for optimal supplier evaluation in a biomass supply network. High degree of time-dependency and uncertainty involved in biomass supply systems renders their management a dynamically evolved issue. The biomass supplier evaluation problem involves conflicting objectives that cannot be optimized simultaneously via a single multiobjective method. A hybrid method based on IFS and multi-periodic optimization was suggested. Our current research intention is to assess more the reliability of decision making by analyzing how sensitive the suppliers' ratings are with respect to the selected criteria and their respective weights.

\section{References}

1. Frombo, F., Minciardi, R., Robba, M., Rosso, F., Sacile, R.: Planning Woody Biomass Logistics for Energy Production: a Strategic Decision Model. Biomass and Bioenergy 33(3), 372-383 (2009)

2. Awudu, I., Zhang, J.: Uncertainties and Sustainability Concepts in Biofuel Supply Chain Management. Renewable and Sustainable Energy Reviews 16(2), 1359-1368 (2012)

3. Atanassov, K.T.: Intuitionistic Fuzzy Sets. Fuzzy Sets and Systems 20(1), 87-96 (1986)

4. Li, D.F.: Multiattribute Decision Making Models and Methods Using Intuitionistic Fuzzy Sets. Journal of Computer and System Sciences 70(1), 73-85 (2005) 
5. Agarwal, P., Sahai, M., Mishra, V., Bag, M., Singhet, V.: A Review of Multi-Criteria Decision Making Techniques for Supplier Evaluation and Selection. International Journal of Industrial Engineering Computations 2(4), 801-810 (2011)

6. Chan, F., Kumar, N., Tiwari, M.K., Lau, H.C.W., Choy, K.L.: Global Supplier Selection: a Fuzzy-AHP Approach. International Journal of Production Research 46(14), 3825-3857 (2008)

7. Wang, J.W., Cheng, C.H., Huang, K.C.: Fuzzy Hierarchical TOPSIS for Supplier Selection. Applied Soft Computing 9(1), 377-386 (2009)

8. Boran, F.E., Genc, S., Kurt, M., Akay, D.: A Multi-Criteria Intuitionistic Fuzzy Group Decision Making for Supplier Selection with TOPSIS Method. Expert Systems with Applications 36(8), 11363-11368 (2009)

9. Ye, F.: An Extended TOPSIS Method with Interval-Valued Intuitionistic Fuzzy Numbers for Virtual Enterprise Partner Selection. Expert Systems with Applications 37(10), 7050-7055 (2010)

10. Szmidt, E., Kacprzyk, J.: Distances between Intuitionistic Fuzzy Sets. Fuzzy Sets and Systems 114(3), 505-518 (2000) 